\title{
Human-driven Machine-automation of Engineering Research
}

\author{
M. D. L. Millen, A. Viana Da Fonseca \& X. Romão \\ Department of Civil Engineering \\ Faculty of Engineering of University of Porto, Portugal
}

\begin{abstract}
This paper presents a framework for efficiently producing engineering research in a global collaborative effort in a rigorous scientific manner. The current state of engineering research is subjective, requires an enormous amount of non-stimulating work and lacks a suitable formal structure for efficient information sharing and collaboration. The proposed framework involves multiple research groups setting up different webservers that can perform the steps of the scientific method and automatically determine the quality and value of new research by directly communicating between servers via public and private application programming interfaces (APIs) using a set of object-oriented protocols. The automation of many mundane research tasks (e.g. data manipulation), would allow researchers to focus more on the novel aspects of their research efforts. The increased clarity around the quality and value of research, would allow the research efforts of individuals and available research funding to be better disbursed. The paper discusses the major aspects of the scientific method, object-orientated programming, the application of the proposed research framework for experimental/analytical/numerical engineering research, some of the potential benefits and drawbacks, as well as the current state of implementation.
\end{abstract}

\section{INTRODUCTION}

A major issue with the current research process is that it requires an enormous amount of effort to stay upto-date with the latest research in a given field. From this issue stem several others including: redundant research (because the author and reviewer were not up-to-date), poorly founded research (the author did not understand the necessary existing literature that supported the new findings), weak research (the new hypotheses provides weaker conclusions than previous research), to name but a few issues. This reflects an unhealthy industry and makes it difficult to perform further research based on the current global understanding, therefore researchers typically build off only their own research and the research of a select few. There is definitely more research being done than in the past, but we cannot 'stand on the shoulders of giants' as efficiently as Isaac Newton.

Historically this has happened before. Mathematical research in the 19th century still relied on human intuition, and in some cases, had inconsistencies and lacked formal proofs that underpinned major branches of mathematics. There was a movement, "Hilbert's program", to rigorously rebuild mathematics from its foundations using a set of axioms to reprove and formalise old theorems (Hilbert 1902). The rebuilding and formalisation of mathematics allowed mathematicians to more easily collaborate and to develop more advanced and consistent theories. Another case is the work of René Descartes (1596-1650), who wanted to remove all doubt from science and philosophy by completely rebuilding it from nothing, the first truth: "I think, therefore I am". Currently, many fields of science are suffering from poorly organised global research and non-reproducible results, which has prompted new initiatives such as the 'Reproducibility Project' in Psychology (Poldrack and Poline 2015). While in other fields of research they have fully formalised the research process and use robots and machine learning to automate scientific discovery for some narrow research problems such as drug development (Sparkes et al. 2010). Engineering research does not need to be as extreme as Descartes or move completely to robotics, but a greater focus on consistency is required now to allow research to happen efficiently and effectively at a global level.

In addition to the poor consideration of consistency, many research fields have changed considerably in the last century, moving from the study of individual mechanisms and phenomena to system-based effects (Foster 2006). The study of complex systems typically requires large simulations with large amounts of data and some knowledge of computational techniques. Djorgovski (2005) argues that applied computer science in research fulfils the role of 
applied mathematics in the 17th to 19th centuries, providing a formal framework for exploring science. The increased complexity of system-based effects research makes it harder to quickly repeat an experiment/simulation or manually check the underlying calculations. To improve the repeatability of new research, one solution would be to present new research in a machine-readable format to allow everyone to re-run the exact same simulation or have a computer cross-check experimental results against other existing results.

Fortunately in the field of earthquake engineering research, there are several initiatives that are greatly improving the situation by open-sourcing their findings and providing machine-readable output from their research (e.g. software or databases). OpenSees (McKenna 2011), an open-source software for structural and geotechnical engineering, allows research to be embedded into OpenSees subroutines, which enables others to easily validate those findings and use them in their own research. The use of exactly the same subroutines across various research projects also provides some level of consistency in that the underlying assumptions of the numerical simulations are the same.

The open-source object-orientated programming language Python, has also grown a large user base of engineering researchers, where standard libraries now exist for site-response analysis, ground motion analysis and unit analysis, among other libraries. This effort has recently been supported with the allocation of Digital Object Identifiers (DOIs) for software packages through Zenodo, a CERN and OpenAire initiative. The DOIs support having multiple versions of a software which "enables users to update the records files after they have been made public and researchers to easily cite either specific versions of a record or to cite, via a top-level DOI, all the versions of a record" - Zenodo (2018). In support of the direct implementation of scientific research into software, frameworks for the evaluation of the quality and predictive capabilities of scientific software have been developed for particular situations (e.g. Oberkampf et al. (2003), Bradley (2011)).

The creation of online experimental and field data repositories (e.g. The Europe SERIES database (University of Patras 2018) or the US Design-Safe-CI database (Rathje et al. 2017)) also enables a reviewer to more easily validate research findings, by downloading the raw data and comparing the results against existing theoretical work or other experimental work. Other databases such as the NGA2 strong ground motion database (Ancheta et al. 2013) and the European strong ground motion database (Akkar et al. 2013), also facilitate the validation of experimental and numerical research in earthquake engineering, where all researchers have the ground motions readily available, making it easier to re-construct the experiment/simulations of existing literature.
However, these advancements are still not nearly enough. The effort required to replicate a numerical study of another researcher often takes months and typically requires requesting additional information not supplied in the original publication. The replication of experimental results or the comparison of two analytical expressions can be equally difficult.

Müller (1958) conceived a solution to these problems for research in the physical sciences: "It would seem eminently feasible, however, to punch program cards for tens of thousands of cards for as many empirical or fundamental equations and the data to which they apply. In a suitable computer center or agency these could be interrogated when necessary and the detailed data sent to a subscriber by teletype or more leisurely by mail." To address these problems in engineering research, this paper presents a framework for implementing the empirical and fundamental equations of engineering research in computer code, similar to Müller's concepts. However, using modern technology thus avoiding the need for teletype and mail!

The framework outlines a process to rapidly improve the validation time of existing research and allow researchers to stay up-to-date with new developments, as well as increasing the quality and value of new research. The major aspects of object-orientated programming and the scientific method are discussed and the proposed research framework is presented along with the current application of the framework to research assessing the earthquake performance of buildings on liquefiable soils.

\section{THE SCIENTIFIC METHOD}

Engineering research is first and foremost a scientific pursuit. It is therefore underpinned by the scientific method, a procedure to develop new knowledge by evaluating the predictive capabilities of hypotheses against measurements from experiments. The exact steps vary for different scientific pursuits, however, the process must develop a falsifiable hypothesis (i.e. there could conceivably be experiment that could prove the hypothesis to be false), and the experiment should test whether the hypothesis is false. Some engineering research can have a different focus than pure science, such as research that is for the practical improvement of a product. Although this research can still follow the scientific method, it is typically better defined by product development methodologies.

In Figure 1 the main steps of the scientific method are outlined and can be described as:

1. Develop a research question. This step is typically driven by curiosity about why something works or from more practical demands.

2. Develop a hypothesis. This is the formulation of a concept that could be used to provide a prediction for the the research question. 


\begin{tabular}{|c|c|c|c|}
\hline $\begin{array}{l}\text { 1) Develop } \\
\text { Research } \\
\text { question }\end{array}$ & $\begin{array}{l}\text { 2) Form testable } \\
\text { hypothesis }\end{array}$ & $\begin{array}{l}\text { 3) Conduct an } \\
\text { Experiment }\end{array}$ & $\begin{array}{l}\text { 4) Analyse data } \\
\text { and compare } \\
\text { with hypothesis }\end{array}$ \\
\hline
\end{tabular}

Figure 1: The steps of the scientific method

3. Conduct an experiment. Collection of data that could prove the hypothesis is incorrect.

4. Analyse data and compare with hypothesis. In this step the comparison between experimental results and the prediction attempts to nullify the proposed hypothesis.

High quality blind-predictions of experimental tests are a clear application of the scientific method in engineering research and provide an objective quantification of the true predictive abilities of current hypotheses. However, given the current difficultly of fully understanding someone else's research, often this process gets performed by a single person or research team, where hypotheses are presented and experiments are performed in a closed group without a comparison to existing hypotheses and data from other groups. An accepted scientific theory should be able to sufficiently explain all prior experimental observations and the best current hypothesis should be the hypothesis that best explains all of the prior experimental observations.

\section{OVERVIEW OF OBJECT-ORIENTED PROGRAMMING}

Engineering research involves the understanding of physical objects and therefore it is well suited to the paradigms of object-orientated programming (OOP). Conceptually OOP allows the representation of physical and conceptual objects and their parameters as numerical objects (i.e. the software can store in memory a building and its parameters (e.g. height, width, floor weights, etc)). The alternative to OOP is typically procedural programming, where a software is written based on functions and subroutines and therefore the parameters do not have additional relationships defined outside of what is performed within the function/subroutine.

The additional structure required for OOP can sometimes be a hindrance, but the structure can also be used to enforce compatibility between different parts of a software and greatly reduce the amount of repeated source code. Figure 2 conceptually shows the difference between the two approaches, where in OOP approach the additional structure of the parameters is stored in memory and can be passed directly into the function to design a building, while the procedural approach requires explicitly passing each parameter to the function and then re-establishing the relationships between parameters inside of the function. The OOP approach is particularly well suited to engineering because parameters typically have many interconnected relationships.

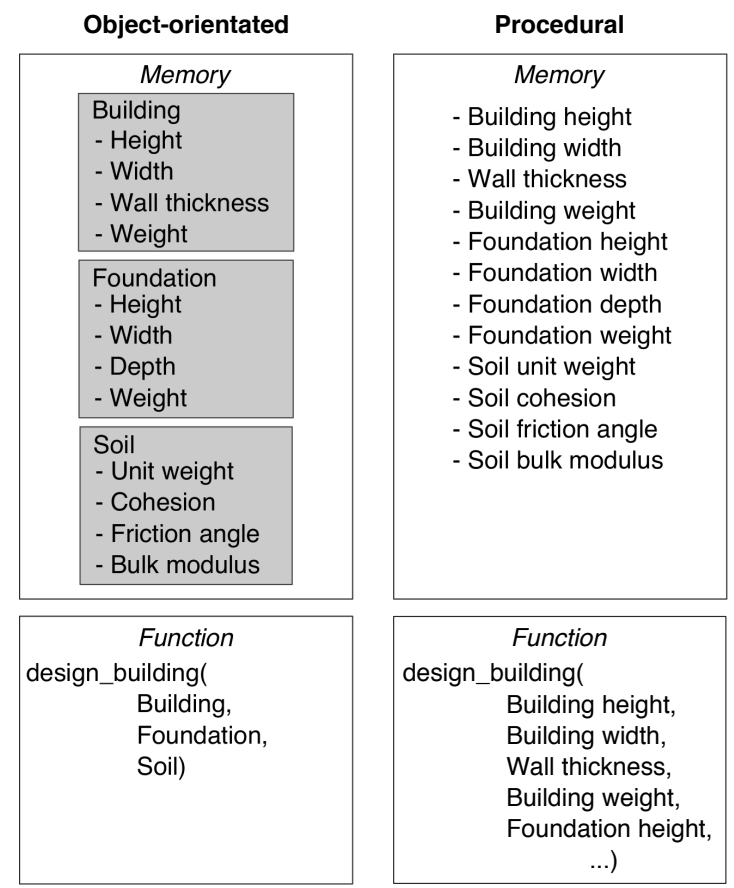

Figure 2: Conceptual comparison between object-orientated programming and procedural programming

When multiple people use the same code it is important that they have the same definition of the parameters and the relationships between them (e.g. is the foundation height measured to the base of the concrete or the lowest layer of the reinforcing steel?). The structure that is inherent when using OOP means that the parameter definitions are tied to the objects and therefore you just need to know if you are using the same object (in Figure 2, this reduces the chance of inconsistent parameters from twelve to three). The structure of OOP is therefore a key aspect to improve collaboration directly at the calculation level.

An integral part of research is the development of new concepts and the adaption of existing concepts for new needs, and therefore flexibility in a research workflow is very important. OOP provides a toolset for extending and adapting existing concepts through a technique called abstraction. Typically in OOP, an object is defined by inheriting parameters from a parent object and then adding the unique parameters specific to that object, while another object would also inherit from the same parent object but have a different set of unique parameters. The process of abstraction is defining which parameters should belong to the parent object and what should be unique. Abstraction is not just used in OOP. In fact it used in many engineering processes and scientific concepts such as the taxonomic ranks in biology. The Tiger species in Figure 3 can be abstracted to the genus Panthera, which includes lions, leopards and jaguars, which all share the same flattish skull among other attributes. The Panthera genus can be abstracted to the family, Cat, which includes all cats, and can be abstracted further to the order, Carnivore, class, Mammal, phylum, Vertebrate, kingdom, Animal. At each abstraction there are a set of common attributes that are shared, and this structure has allowed researchers to easily communicate 


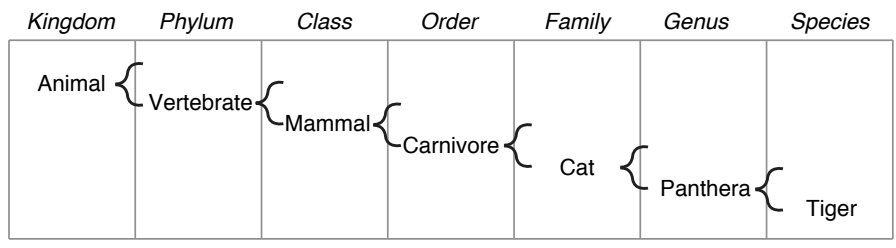

Figure 3: Abstraction applied to the taxonomic ranks of the Animal Kingdom

the attributes of new biological forms without having to explain all of the features but by simply stating what genus it is from and defining the unique characteristics at the species level.

For engineering research a series of base objects (e.g. soil, foundations, beams and columns) could therefore be extended to cover the unique cases of new research. When objects inherit from the same parent they have parameters that are common and, in some cases, can be used interchangeably by other functions and objects. It is this attribute of OOP that allows research to be developed with consistency.

\section{A HUMAN-DRIVEN MACHINE-AUTOMATION RESEARCH PROCESS}

The framework proposes implementing all of the existing engineering hypotheses and experimental and field data into computer readable form in a way that allows it to be automatically crossed checked for consistency, similar to the concepts of Müller (1958). The experimental data and hypotheses could exist on a distributed set of web-servers ("Müller machines"), that would expose application programming interfaces (APIs) to receive input parameters as objects and return objects or values that represent results of an experiment or the prediction from a hypothesis. A research question could be defined on a web-server using objects and a set of criteria to evaluate the accuracy (see Section 4.4), thus initiating a automated research workflow. The web-server would then check available APIs to see whether they can provide experimental data or predictions.

A path finding algorithm could also be employed to formulate a prediction by connecting the inputs and outputs of multiple hypothesis to provide the required outputs from the given inputs. Figure 4 shows how multiple hypotheses could be pulled together to provide a prediction for building damage in terms of foundation settlement, column damage and beam damage based on a set of inputs (Earthquake fault, Building location, Soil profile, Foundation, Building). Each hypothesis shown in Figure 4, could be formulated by many smaller element- or mechanismlevel hypotheses and each hypothesis could be validated at the element level and at the whole system level against available experimental data sets. There could potentially be many different combinations of hypotheses that could provide a prediction. In the example in Figure 4 the hypotheses could be consider-

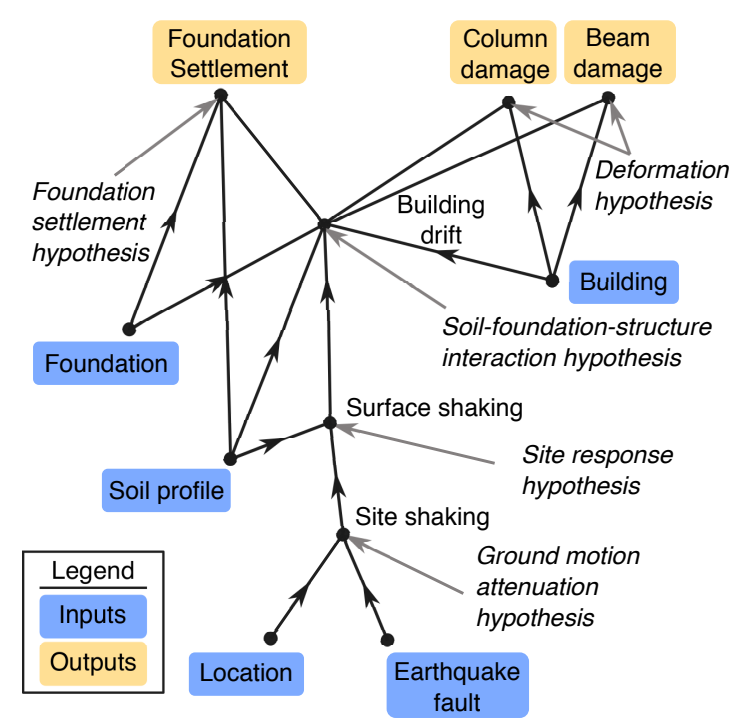

Figure 4: A prediction of building damage parameters from objects

ing the ground shaking as a time-series or as a spectral quantity. Different set of hypotheses could be used that consider the influence of the building's torsional response or ignoring the influence of the site response or soil-foundation-structure interaction. All possible combinations that take the required inputs and produces an estimation could be scored (based measures of level of uncertainty, bias, physical insight, etc) to provide a scorecard of the current knowledge for answering a given question. Limitations could also be imposed on the range and use of particular hypotheses to avoid excessive extrapolation of trends. The research question could remain an active question, such that as more experimental data becomes available and new hypotheses are developed, they are automatically assessed against the existing experimental data and hypotheses.

In the following sections the main aspects of the framework are outlined, the application to analytical and experimental research is explained and the role of scoring functions is explored. The final section discusses the current implementation of the framework for research on the earthquake performance of buildings on liquefiable soil, as part of the LIQUEFACT H2020 research project.

\subsection{Main aspects}

The framework is intended to improve the quality and value of new research in an efficient and easy to understand manner. To achieve this, the following philosophies have been adopted:

1. Follow the scientific method. Question $\rightarrow$ Hypothesis $\rightarrow$ Experiment $\rightarrow$ Comparison

2. Extendable. The project allows the development and connection of new yet-to-be-conceived research

3. Convention over Configuration. The benefits of a convention (e.g. standard format for presenting 
experimental data) out-weigh the benefits of infinite configurability

4. Repeatable research. Clear documentation of new research such that it could be repeated by an equally equiped and skilled researcher

5. Human readable. The reasoning behind a hypothesis must be interpretable by a human

6. Open-source research. Research should be provided open-source to allow others to use and validate it

Note that this list does not include common research goals of accuracy, physical insight, simplicity of description or application. However, these are important attributes of high quality engineering research and they will be dealt with in Section 4.4 on scoring functions, where the quality of research is discussed. Also note that this framework does not require the use of artificial intelligence or neural networks. In fact obscure processes such as neural networks often struggle to fulfil the main steps of the scientific method of having repeatable results and a clear line of reasoning. While they can be helpful for finding trends and formulating a hypothesis, similar to the role of human intuition, if they are applied on their own for predictive purposes, they can be considered a completely different approach to developing knowledge.

\subsection{Application for experimental, field and long numerical simulation data}

Experimental and field data is often collected to evaluate a hypothesis and is a key part of the scientific method. More recently numerical simulations are being used to evaluate hypotheses, in the same role as experimental data. Therefore, numerical simulations can fulfil two roles in the scientific method: a pseudo experiment (i.e. can a simplified method (hypothesis) capture the behaviour of the numerical simulations (experiment)), as well as being a hypothesis (can the numerical model simulate the experimental behaviour). In the proposed framework short opensourced numerical procedures can be considered as hypotheses, which could be used to provide a prediction for new experiments, while long numerical simulations using commercial software cannot easily be applied to new data sets and therefore only fulfil the role of a pseudo experiment.

One of the key parts of experimental research is the comparison of a data set with existing hypotheses and other data sets. This comparison not only provides a level of validation of the data set but it provides useful insights into unique intricacies that were recorded in the new or existing data sets. This comparison is essentially data processing and typically involves a large amount of mundane manual work. This framework aids the comparison, where standard input and

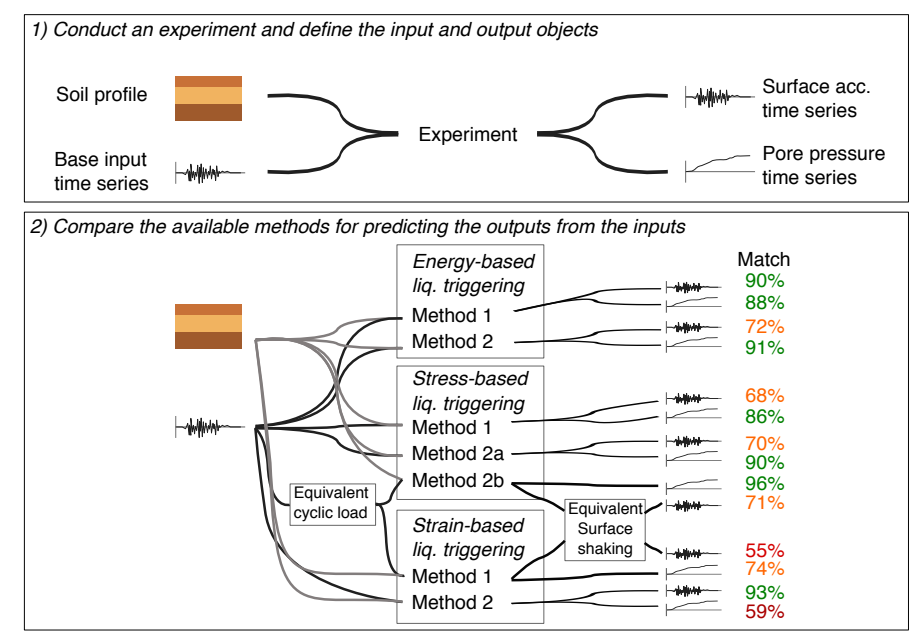

Figure 5: Conceptual application of the engineering consistency project for liquefaction triggering analysis

output files are written for each experiment, which define the measured input and output objects using a language agnostic format such as json or yaml (Figure 5 - step 1). The new data set is then uploaded to an online database and the standard input and output files would be submitted to a web-server, where an API would automatically be built for the dataset. The web-server could then compare the experimental results against all existing data sets and against all existing relationships (Figure 5 - step 2). The web-server could easily produce the required plots of the comparisons and build the appropriate references, ready for journal publication.

\subsection{Application for analytical and empirical research}

Analytical research is referred to here as the development of new hypotheses based on the adaption of existing hypotheses using mathematical reasoning. Empirical research is referred to here as the development of new hypotheses based on the fitting of a curve through a data set using statistical regression. Typically new hypotheses in engineering are developed through a combination of both analytical and empirical techniques.

A new hypothesis is typically developed to answer a specific research question (e.g. estimating liquefaction triggering (Boulanger and Idriss 2014)). The new hypothesis is then compared to existing less complex situations (e.g. elastic solutions) and experimental/field data or numerical simulations that are independent of the data set that was used to originally develop the hypothesis. This comparison process is an important part of validating a new hypothesis but it can take an enormous amount of effort to re-interpret independent data sets, especially if they are in different formats.

The framework aids this validation, where the new hypothesis can be written into an OOP language in which the inputs and outputs are compatible with a standard set of objects. The new equation is then sub- 
mitted to a web-server where an API is generated for it to receive the input objects and output the predictions. The web-server then automatically compares the new hypothesis against all existing data sets and against all exist hypotheses (step 2 in Figure 5).

This process would provide an independent and objective 'score' of the validity and value of new research, as well as highlight inconsistencies between hypotheses. Completely independent validation is a key role of the reviewer for publication, and therefore the review process for publication could be greatly reduced.

\subsection{Scoring functions}

The framework could provide an independent evaluation of the quality and value of research. However, this requires an objective quantification of quality and value.

Research quality is largely focused on accuracy. For experimental work, accuracy is difficult to completely establish and typically relies on an honest account from the experimental researcher or a repeated study. For new hypotheses the accuracy can be directly measured through validation and verification procedures that quantify uncertainty and error for given validation metrics. Oberkampf et al. (2003) outlines the key steps in the validation and verification procedure for assessing computational physics software, highlighting that the specification and use of validation metrics is the most important part of the validation process. Validation metrics are the measures of the response that can be used to evaluate the difference between the experimental result and the prediction from the simulation. The choice of metrics is not a trivial step, and is typically highly dependent on the end-use of the prediction, where metrics should be used that allow the minimisation of the uncertainty for the end-use. Therefore for a given set of experiments, and given set of analytical/empirical/numerical predictions, the accuracy would be dependent on the end-use. As an example, for the seismic response of a soil deposit, the enduse case may be the seismic response of a building and therefore the energy of the surface motion in the frequencies closest to the natural frequencies of the building are the most important metrics, while if the end-use was the estimation of design loads on a buried pipeline, then the soil strains and displacements at the depth of the pipe are of greatest interest.

Bradley (2011) outlines a framework for the validation of constitutive models for modelling the seismic response of a soil deposit, suggesting the use of a vector of engineering demand parameters for the validation metrics (e.g. peak ground acceleration, displacement and Arias intensity). Generally engineering demand parameters offer a useful tool for validation metrics as they tend to be quantities that are of interest for a wide-variety of end-use applications and they are

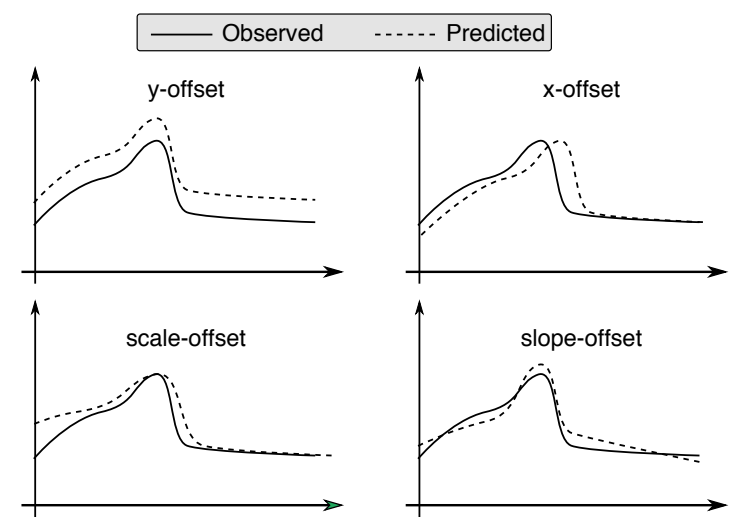

Figure 6: Several types of errors for 2 dimensional data

a single quantity. However, some measures are two dimensional (e.g. pore-pressure build up versus time) or even three-dimensional (inter-storey drift versus height versus time). The error and uncertainty associated with two-dimensional (and three-dimensional) parameters have additional considerations compared to a single value due to their inter-dependence. Simply computing the difference in the two dimensions (e.g. X-offset and y-offset in Figure 6) may fail to capture the accuracy of the prediction which may be offset by scale or slope. Further work is required to develop standard validation metrics. However, for initial implementation purposes the researcher who asks the initial question (Step 1 of the scientific method) would also define the validation metrics.

The measure of research value for field studies and experimental work can be directly quantified by the reduction in the uncertainty of existing hypotheses (or of the null hypothesis) especially for previously untested ranges of input parameters. Also repeating an existing experiment can offer immense value by reducing the uncertainty around the experimental results. The measure of research value for a new hypothesis, especially in engineering research, should be concerned with attributes such as physical insight, simplicity in application and sufficient generality as outlined by (Wolf and Deeks 2004), as well as considering end use applications. The attributes could be quantified by:

- Physical insight - Difficult to quantify as it is concerned with the ability to communicate the inner workings of the physical problem, but could be evaluated by assessing the number of parameters that have a physical meaning.

- Simplicity in application - Quantified by the number of input parameters or number of calculations or ease of determining the inputs.

- Generality of application - Quantified by the extent of the domain of inputs where the hypothesis could provide a prediction (linked to a level of sufficient accuracy)

- End use application - Could be quantified based 


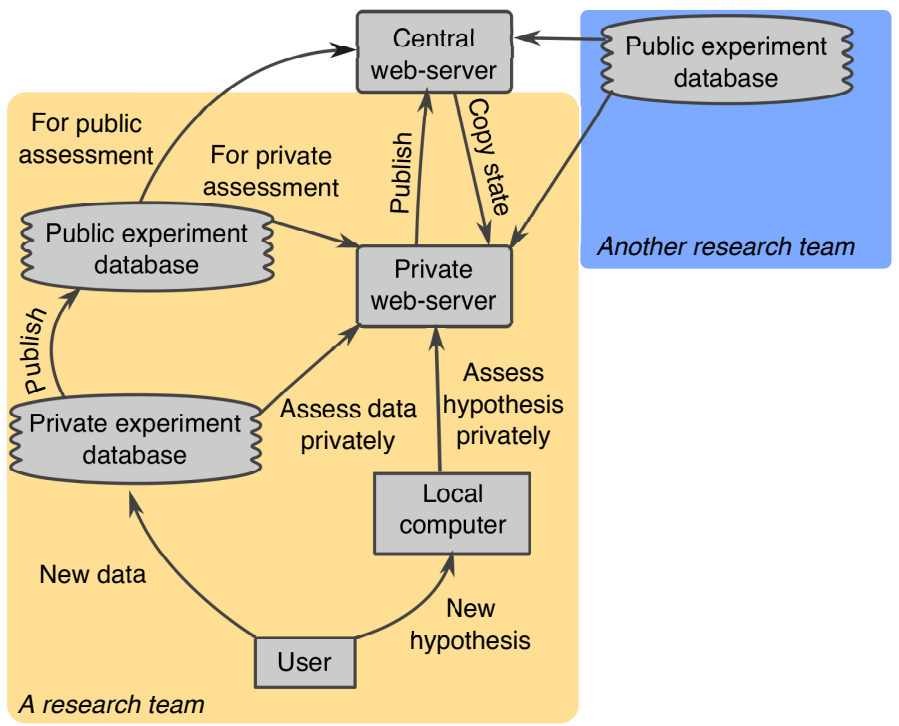

Figure 7: Proposed server and database architecture for the research framework

on the number of predictions that it can be part of for a given set of research questions.

By quantifying the quality and value of all research the framework could quantify the current state of understanding and it would continuously update with the addition of new hypotheses and data sets.

\subsection{Implementation}

The implementation of the framework would provide a flexible system that allows research teams to have full control of their research process and the publication of their data and hypotheses. However, it requires some level of understanding of OOP and webservers as well as the basic concepts of the scientific method. To achieve ease the implementation, the webserver and database architecture could be designed as in Figure 7. A user develops new hypotheses on their local computer, and then can submit the hypothesis to a private server that copies the current state of the public web-server and performs the comparison of the new hypothesis against all of the publicly available data sets and all existing hypotheses. The user can then publish the new hypothesis to the central webserver where it becomes part of the existing hypotheses available for others to use and forms part of the current state of knowledge. Experimental data could be held privately in a private database (in fact in the same database as the public data, just with a private database address), to allow researchers to run comparisons prior to publishing the address and making the data public.

The central web-server allows anyone to ask a research question, which would involve setting the validation metrics, and then the available data sets would be queried and available hypotheses would be compiled and compared to provide an answer to the question.

\subsection{Benefits}

The presented framework offers many benefits to the researcher and the research community:

- Repeatable research. The comparisons would be performed independently by the server, therefore they can be requested by any end-user.

- Updatable. A researcher can update their hypothesis after publication by incrementing a version number, and each version would be stored but they could select which one should be used as the default.

- Quantitative importance. The framework could directly calculate the improvement that a new analytical expression provides to the estimation of an output compared to existing literature. It could also quantify how much a new experimental data set improves validation of existing theories. This would reduce the amount of redundant research and could even highlight to end-users where experiments are needed for validation and where existing hypotheses are weak.

- Automation of recurrent work. The structure applied to research data allows tools to be built that can automate the generation of research outputs such as tables, figures, references, methodologies and many other recurring aspects of research. Potentially the only aspects that cannot be automated are the unique aspects of the research.

- More citations. The paper references (DOIs) to individual hypotheses or data sets can be embedded into the source code or objects that they generate. Thus, when someone publishes a comparison using the framework, the references could automatically be included in the new paper.

- Connect to industry. Research could easily connect to object-oriented software platforms for engineering and architecture (e.g. Building Information Modelling, BIM).

\subsection{Drawbacks}

The presented framework potential contains several drawbacks to the researcher and the research community:

- Exposure to criticism. The exact implementation of a set of equations and the experimental data sets are made publicly available and therefore could more easily be scrutinised

- Effort/Learning. There is an effort involved in learning enough about OOP to implement and make use of the framework 
- Excessive references. Because the framework would test for consistency against all available research, there would then be a large amount of references for each new publication. However, as more research is produced in a particular field, it could be expected that a proper literature review would result in increasing amounts of references. The approach to referencing work might need to change to allow this, where research outputs could be built using a blockchain that clearly stated the exact inputs, equations and data sets that were used to generate the output.

- Gaming of the system. Researchers may focus only on research that would result in a high score (quality and value), while this is the intended purpose of quantifying the quality and value of research, it could misalign research if the scoring failed to recognise the importance of different types of research.

\subsection{Current state}

The goal of the project is large and open-ended, because new theories/hypotheses are continuously being developed. The first step is to focus on the area of engineering research related to the response of buildings on liquefiable soil as part of the LIQUEFACT project. The major hypotheses around liquefaction triggering, building settlement, site response analysis, building response, soil-foundation-structure interaction, are being implemented into Python using a standard set of objects (models for Soil, Foundation, Building, TimeSeries, etc.). The current version of these implementations are publicly available at https://github.com/eng-tools.

\section{CONCLUSION}

The current approach to engineering research typically results in minimal collaboration across research teams, it requires a large amount of manual effort and lacks a consistency approach to validate new hypotheses. This paper presents a more rigorous approach to the application of the scientific method to engineering research. The presented approach takes advantage of web-server technology and object-oriented programming to provide a consistent, structured and continuously updating state of knowledge. The key use cases as well as the potential advantages and drawbacks of the procedure are highlighted to emphasise that the new approach could rapidly speed up the development of new high quality and valuable research. The imposed structure and consistency of the approach, as well as the ability to automatically update the state of knowledge provides the necessary foundation to efficiently develop research at a global level both now and into the future.

\section{ACKNOWLEDGEMENTS}

This paper was produced as part of the LIQUEFACT project (Assessment and mitigation of liquefaction potential across Europe: a holistic approach to protect structures / infrastructures for improved resilience to earthquake-induced liquefaction disasters) has received funding from the European Union's Horizon 2020 research and innovation programme under grant agreement No GAP-700748.

\section{REFERENCES}

Akkar, S., M. A. Sandıkkaya, M. Şenyurt, A. Azari Sisi, B. Ö. Ay, P. Traversa, J. Douglas, F. Cotton, L. Luzi, B. Hernandez, and S. Godey (2013). Reference database for seismic ground-motion in europe (resorce). Bulletin of Earthquake Engineering 12(1), 311-339.

Ancheta, T. D., R. Darragh, J. P. Stewart, S. Emel, W. Silva, B. Chiou, K. E. Wooddell, R. W. Graves, A. R. Kottke, D. M. Boore, T. Kishida, and J. Donahue (2013). Peer nga-west2 database. Technical Report 03.

Boulanger, R. W. and I. M. Idriss (2014). Cpt and spt based liquefaction triggering procedures. Technical report.

Bradley, B. A. (2011). A framework for validation of seismic response analyses using seismometer array recordings. Soil Dynamics and Earthquake Engineering 31(3), 512-520.

Djorgovski, S. G. (2005). Virtual astronomy, information technology, and the new scientific methodology. arXiv.org, 125132.

Foster, I. (2006, mar). 2020 computing: A two-way street to science's future. nature.com 440(7083), 419-419.

Hilbert, D. (1902). Mathematical problems. Bull. Amer. Math. Soc., 429-437.

McKenna, F. (2011). Opensees: a framework for earthquake engineering simulation. ieeexplore.ieee.org 13(4), 58-66.

Müller, R. H. (1958). Computer center for basic physical science data proposed. Analytical Chemistry 30(8), 55A-55A.

Oberkampf, W. L., C. Hirsch, and T. G. Trucano (2003). Verification, validation, and predictive capability in computational engineering and physics. Technical report, Sandia National Laboratories (SNL), Albuquerque, NM, and Livermore, CA.

Poldrack, R. A. and J.-B. Poline (2015). The publication and reproducibility challenges of shared data. Trends in Cognitive Sciences 19(2), 59-61.

Rathje, E. M., C. Dawson, J. E. Padgett, J.-P. Pinelli, D. Stanzione, A. Adair, P. Arduino, S. J. Brandenberg, T. Cockerill, C. Dey, M. Esteva, F. L. Haan Jr., M. Hanlon, A. Kareem, L. Lowes, S. Mock, and G. Mosqueda (2017). Designsafe: New cyberinfrastructure for natural hazards engineering. Natural Hazards Review 18(3), 06017001.

Sparkes, A., W. Aubrey, E. Byrne, A. Clare, M. N. Khan, M. Liakata, M. Markham, J. Rowland, L. N. Soldatova, K. E. Whelan, M. Young, and R. D. King (2010). Towards robot scientists for autonomous scientific discovery. Automated Experimentation 2(1), 1-11.

University of Patras (2018). Series database. http://www.dap.series.upatras.gr/default.aspx. Accessed: 2018-01-20.

Wolf, J. P. and A. J. Deeks (2004). Foundation Vibration Analysis: A Strength of Materials Approach (1 ed.). ButterworthHeinemann.

Zenodo (2018). Zenodo now supports doi versioning! http://blog.zenodo.org/2017/05/30/doi-versioninglaunched/. Accessed: 2018-01-20. 